\title{
Performances Digitais dos Pesquisadores na Iniciação Científica
}

\author{
Digital Performances of Researchers in Scientific Initiation
}

Karine Freitas Souza ${ }^{1}$, Victor Azevedo do Amaral ${ }^{2}$, Isaac de Santana do Santos ${ }^{3}$, Jonatas Alfa Torquato de Souza ${ }^{4}$ e Kisia Souza Santos ${ }^{5}$

1,2,3,4,5 Universidade Federal da Bahia, UFBA 


\title{
Resumo
}

A COVID-19 implicou transtornos na realização da pesquisa intitulada "As mulheres, graduandas e o mar: rede informacional e documental para aposentadoria das marisqueiras" desenvolvida por estudantes de Secretariado Executivo, pelo Programa Institucional de Bolsas de Iniciação Científica [PIBIC] financiado pelo Conselho Nacional de Desenvolvimento Científico e Tecnológico [CNPq]. Os pesquisadores passaram a trabalhar na modalidade remota a fim de concluir a investigação citada. O objetivo do estudo foi conhecer as performances dos pesquisadores na modalidade de trabalho remoto durante a Iniciação Científica. As bases teóricas da pesquisa incluem os estudos da área Secretarial e demais investigações sobre teletrabalho/ trabalho remoto no Brasil. O objetivo foi apresentar o processo de reestruturação metodológica e replanejamento do trabalho dos pesquisadores ocorridos durante a pesquisa citada. A pesquisa é exploratória e a metodologia utilizada foi descritiva qualitativa considerando os relatos de experiências da equipe. Os resultados indicaram êxito na execução dos levantamentos bibliográficos, documentais e na realização das entrevistas e reuniões, todos realizados na modalidade online.

Palavras-chave: iniciação científica, performance digital, teletrabalho, secretariado executivo

\begin{abstract}
The COVID-19 pandemic led to limitations in the accomplishment of the research project "Women, graduating and the sea: informational and documentary network for the retirement of fisherwomen" developed by students of Executive Secretarial Studies, held at the Institutional Program of Scientific Initiation Scholarships [PIBIC] which is financed by the National Council for Scientific and Technological Development [CNPq]. The research team started working remotely to complete the abovementioned research. This study aimed to assess the performances of the researchers working remotely during their Scientific Initiation. The theoretical basis of the research includes studies completed within the Secretarial Studies field and other research works about teleworking in Brazil. The objective was also to present the methodological restructuring and replanning process that occurred during the research period. Having the reports of the team experiences as a main basis, a descriptive-qualitative methodology was adopted. The results indicated a successful accomplishment of bibliographic and documental surveys, as well as of interviews and online meetings.
\end{abstract}

Keywords: scientific initiation, digital performances, telework, executive secretarial studies 
A pandemia do novo coronavírus provocou a adoção de medidas de isolamento social recomendadas pela Organização Mundial de Saúde [OMS] e seguidas pela Universidade Federal da Bahia [UFBA]. Como em outras instituições, a universidade suspendeu as atividades acadêmicas presenciais (Portaria n 103/2020), a partir da promulgação da Lei $n^{\circ}$ 13.979, de 6 de fevereiro de 2020, considerando:

A Declaração de Emergência em Saúde Pública de Importância Internacional pela Organização Mundial da Saúde - OMS, em 30 de janeiro de 2020, em decorrência da Infecção Humana pelo novo coronavírus (COVID-19);

A Portaria n 188/GM/Ministério da Saúde, de 4 de fevereiro de 2020, que Declara Emergência em Saúde Pública de Importância Nacional (ESPIN), em decorrência da Infecção Humana coronavírus;

A Portaria n 356/GM/Ministério da Saúde, de 11 de março de 2020, que regulamenta a Lei n 13.979, de 06 de fevereiro de 2020, que dispõe sobre as medidas que poderão ser adotadas para enfrentamento da emergência de saúde pública de importância internacional decorrente do coronavírus; [...] (Portaria no 103, 2020).

Assim, a referida Portaria da UFBA deliberou pela suspensão das atividades acadêmicas e administrativas, por tempo indeterminado. Além disso, a Universidade em nota explicou que:

Entre as atividades que devem ser paralisadas, podemos listar as atividades curriculares de campo; os concursos e os editais de monitoria, extensão e assistência estudantil; as chamadas de matrícula do SiSU, progressão BI/CPL e ingresso de indígenas, quilombolas e aldeados, refugiados e pessoas trans; e o funcionamento das bibliotecas e da creche da UFBA, bem como dos locais de atendimento ambulatorial e realização de exames ordinários, entre outros. (Universidade Federal da Bahia, 2020).

O Programa de Iniciação Científica sob coordenação da UFBA também sofreu com a suspensão das atividades, uma vez que todas as de campo estariam suspensas conforme a Portaria. Desse modo, a COVID-19 demandou criação e aumento do trabalho remoto impondo desafios nos usos das técnicas, para efeitos deste estudo, das técnicas de pesquisa e das técnicas secretariais; identificação das tecnologias disponíveis e maior letramento digital dos trabalhadores. Também requereu habilidades sociais para questões relacionadas às emoções, comunicação, motivação, negociação, dentre outros fenômenos correlatos.

Nesse contexto, os alunos de Secretariado Executivo que participam de uma pesquisa de Iniciação Científica intitulada "As mulheres, graduandas e o mar: rede informacional e documental para aposentadoria das marisqueiras", pelo Programa Institucional de Bolsas de Iniciação Científica [PIBIC] e financiada pelo Conselho Nacional de Desenvolvimento Científico e Tecnológico [CNPq], ficaram impossibilitados de ir a campo para realizar entrevistas presencialmente e coletar outros dados, como fotos das trabalhadoras no exercício do ofício. Surgiram também demandas por novas habilidades e competências infocomunicacionais para o desenvolvimento do trabalho de pesquisa remoto.

O teletrabalho é definido pela Organização Internacional do Trabalho [OMT] como uma modalidade de trabalho realizada distante do escritório ou um centro de produção, separação física que demanda o uso de 
tecnologias que sejam facilitadoras da comunicação. A modalidade está em crescimento no país (Matos, 2016; Sociedade Brasileira de Teletrabalho e Teleatividades [SOBRATT], 2020) e sua regulamentação foi normatizada no ano de 2017 com a Reforma Trabalhista.

De fato, com o advento da internet e a popularização dos notebooks houve crescimento do teletrabalho no Brasil - verificado nos últimos 20 anos. Além disso, a pandemia da COVID-19 demanda novas possibilidades de trabalho remoto e exige novas habilidades. $O$ trabalho remoto apresenta desafios que se relacionam às técnicas, tecnologias disponíveis; letramento digital dos trabalhadores (especialidades nas hard skills) e requer habilidades sociais para questões relacionadas a emoções, comunicação, motivação, negociação, dentre outros fenômenos correlatos (soft skills). Em termos históricos, o trabalho remoto via internet foi tratado pela primeira vez por Nilles (1973).

Para além da situação do trabalho remoto nos últimos anos no Brasil, é preciso considerar que a Lei № 13.467, de 13 de julho de 2017, normatizou o regime jurídico do teletrabalho (Lei no 13.467, 2017). E mais recentemente, com a pandemia da COVID-19, a Lei № 13.979, de 6 de fevereiro de 2020 - impôs Medidas sanitárias (Lei no 13.979, 2020); por sua vez, o Decreto Legislativo no 6, 20 de março de 2020 - declara estado de calamidade pública nacional em especificidades que incidiram sobre as relações de trabalho subordinado (Decreto Legislativo no 6, 2020). Consoante, a Medida Provisória no 927, de 22 de março de 2020, orienta sobre: i) preservação do emprego e da renda; e para ii) o enfrentamento do estado de calamidade pública e da emergência de saúde pública de importância internacional determinam as práticas de vida e trabalho em decorrência da COVID-19 (Medida Provisória no 927, 2020).

A SOBRATT, a Fundação Getúlio Vargas [FGV] e o Serviço Brasileiro de Apoio às Micro e Pequenas Empresas [SEBRAE] desenvolveram estudos sobre os casos brasileiros mais emblemáticos na esfera pública: o Serviço Federal de Processamento de Dados [SERPRO] (2020), que atua com a modalidade de teletrabalho desde 2006, e o Tribunal de Contas da União, que adotou o trabalho remoto em 2012. Em decorrência da necessidade do trabalho remoto por força de saúde pública, surgiram manuais e cartilhas cujos conteúdos tratam das técnicas, tecnologias, direitos e deveres dos trabalhadores remotos. Em geral, essas cartilhas e manuais recomendam sobre: ergonomia, vestuário, comportamento e cenário, entre outros. Salientam condições de trabalho que mantenham a saúde mental do trabalhador remoto.

Para melhor compreensão da questão deste estudo, recorremos às bases teóricas que incluem os estudos da área Secretarial realizados por Fanzeres e Lima (2018), incluímos Rosenfield e Alves (2011) ao refletirem sobre a autonomia e o trabalho informacional, referiram-se o teletrabalho como um elemento das mudanças organizacionais estratégicas que apontam novas formas de trabalho flexível sustentadas por TICs. Todavia, não desconsideram fatores como atipicidade, flexibilidade ou até mesmo a possibilidade de precarização que existe em algumas atividades. A autonomia, aqui conceptualizada sob a ótica enciclopédica e não no sentido filosófico para o termo, a priori evidencia a autodeterminação dos trabalhadores, suas responsabilidades e liberdades para lidar 
com as situações previstas e imprevistas, controlando o ambiente e as etapas do processo de desenvolvimento da atividade.

Os usos das técnicas secretariais foram de notável importância, vistos os riscos que acompanham a coleta de dados na internet quanto ao fenômeno intitulado de infodemia, descrito por Arroyo-Sanchez (2020) como notícias amplamente difundidas e não amparadas pelos saberes científicos, com o objetivo da desinformação e instauração do medo irracional com efeito desestabilizador na sociedade. Compreender esse fenômeno foi fundamental para análise das fontes das informações e coleta de dados nas entrevistas realizadas, bem como, no processo de aprimoramento das técnicas secretariais nos usos das hard skills.

Em complemento, consideramos também recentes pesquisas sobre o teletrabalho no Brasil da SOBRATT (2020), onde diz que ele já era uma realidade para mais de 15 milhões de brasileiros, conforme os dados que seguem:

- $\quad$ Crescimento de $22 \%$ do home office entre 2016 e 2018;

- Pesquisa foi realizada com mais de 300 empresas de diferentes segmentos e portes, com capital nacional e internacional, que empregam mais de 1 milhão de pessoas;

- $\quad 45 \%$ das empresas já praticavam home office e $15 \%$ avaliavam a implantação;

- $\quad$ Segmentos com maior representatividade (44\% do total) TI/telecom (28\%) e serviços (16\%). Áreas: tecnologia da informação, recursos humanos marketing, controladoria/finanças e jurídico (SOBRATT, 2020).

De acordo com Fanzeres e Lima (2018, p. 59), é importante "uma qualificação que suporte a constituição de uma identidade profissional diferente da tradicional, em que os diversos fatores constituintes do sucesso desse tipo de trabalho sejam aprimorados", o que foi confirmado neste estudo.

Diante do isolamento em decorrência da COVID-19, os pesquisadores, graduandos do curso de Secretariado Executivo da UFBA, se depararam com a questão: Quais as performances dos pesquisadores da Iniciação Científica na execução de pesquisa na modalidade de trabalho remoto/teletrabalho?

Para tanto, foi preciso identificar as necessidades dos pesquisadores e como eles reestruturaram a metodologia das atividades de pesquisa de um projeto PIBIC adaptando-a para o trabalho na modalidade virtual. As mudanças incluíram investigar, testar e utilizar plataformas e aplicativos comunicacionais de uso remoto. Elencar alternativas que não onerassem os discentes, e utilizar as técnicas secretariais aprendidas no curso de Secretariado Executivo da UFBA. Foi deste modo que os pesquisadores PIBIC puderam realizar diversas atividades e inclusive participar de evento científico internacional.

\section{Metodologia}

Trata-se de uma pesquisa exploratória uma vez que averigua questões relativas ao desempenho dos pesquisadores PIBIC, no ano de 2020, frente à intempestividade da COVID - 19. Possui caráter documental no uso da técnica de coleta de dados, pois foram levantadas suportes teóricos e informações necessárias para consecução dos objetivos. É também um estudo qualitativo de acordo com a abordagem do problema, pois o propósito 
visou conhecer as performances dos graduandos de Secretariado Executivo da UFBA participantes do PIBIC, no desenvolvimento das atividades de Iniciação Científica, utilizando os seus relatos de experiências com o trabalho remoto. Ao todo foram entrevistados e acompanhados os relatos de cinco estudantes, pesquisadores PIBIC.

O recorte temporal considerou o início do isolamento social na cidade de Salvador, Bahia, de março a maio de 2020, e à interrupção das atividades presenciais pela UFBA. Foram identificadas as ações, competências e habilidades adquiridas/desenvolvidas na graduação em Secretariado Executivo posteriormente aplicadas na realização da pesquisa de iniciação científica, na modalidade remota. A pesquisa considerou os conhecimentos e as experiências dos pesquisadores adquiridas com as disciplinas cursadas na graduação, de modo a dar suporte no planejamento das ações operacionais. Em especial, destacamos os conhecimentos advindos das disciplinas "Competência Informacional", da Ciência da Informação, conteúdo exclusivo da UFBA. Além das disciplinas de "Metodologia Científica" e "Técnicas Secretariais".

Na concepção de Belluzzo (2018), a aplicabilidade das competências em informação no cotidiano das pessoas é decorrente de um processo de interação continuada e internalização de conceitos, atitudes e as tratativas dadas à busca e seleção da informação, de modo a compreendê-la quanto a sua abrangência.

\section{Resultados e Discussão}

Verificou-se que as técnicas secretariais foram utilizadas para o planejamento do trabalho, a realização de reuniões, os treinamentos da equipe, a elaboração de instrumentos de entrevistas e debates na modalidade online. As principais técnicas empregadas foram: planejamento de rotinas e gestão do tempo; elaboração e follow up de agenda e tarefas; organização de reuniões on-line (incluindo convites e confirmações); elaboração e correção de documentos (relatórios e textos), elaboração de cartas-convites e cartas-agradecimentos pelo grupo em pasta compartilhada pelo Google Drive.

Durante a pesquisa diversos documentos foram criados para atender às demandas de ordem científica (artigos, pôster - escrito e virtual) além da elaboração da cartilha, produto final da pesquisa (com ISBN), tanto para modalidade física quanto virtual, e que será disponibilizada para sociedade em fevereiro de 2021 durante o Congresso UFBA. A pesquisa contou com todo o trabalho realizado desde março 2020 até o seu final, em julho de 2020, na modalidade totalmente on-line, de forma colaborativa entre os participantes PIBIC.

A disposição das tecnologias digitais, sobretudo as tecnologias de informação, tornou-se possível realizar reuniões na modalidade virtual entre os pesquisadores e a coordenadora do projeto, através de aplicativos e plataformas gratuitas de videoconferência, a fim de manter o desenvolvimento do estudo e as ações conforme o calendário proposto no projeto. Não obstante, no processo de reestruturação do modo de fazer a pesquisa foram mantidas as atividades constantes no Projeto a fim de consolidar as bases teóricas e informacionais dos pesquisadores.

As adaptações pensadas e aplicadas pelo grupo se basearam na busca dos meios para consecução da pesquisa PIBIC. Os alunos participantes compreenderam que o trabalho dependia de uma performance adequada à nova modalidade, completamente virtual. Para tanto, os discentes planejaram buscar meios de trabalho que 
considerassem: 1) gratuidade da plataforma; 2) facilidade de acesso; 3) praticidade de uso; 4) suporte para salas virtuais com muitas pessoas conectadas simultaneamente; 5) consumo inteligente de pacote de dados.

A primeira consideração do grupo avaliou a possibilidade de adquirir serviços prestados pelo aplicativo ou plataforma sem que houvesse a necessidade de investimento de ordem financeira, e que este não fosse um fator limitador para o alcance da pesquisa. A segunda consideração observou o aspecto da facilidade de acesso, ou seja, quais as formas que os participantes poderiam acessar a ferramenta. Optou-se pela praticidade de instalação em dispositivos móveis, sendo estes mais populares que desktops e notebooks e mais acessíveis para os estudantes partícipes. O terceiro ponto levou em conta o letramento, habilidades e competências informacionais e tecnológicas digitais dos usuários. Para tanto, em atenção a esta categoria foram escolhidos os meios com interfaces simples e básicas nas suas funções evitando, assim, desconfortos durante o uso, caso não houvesse familiaridade com o sistema. Não obstante, o quarto quesito para a escolha do sistema considerou os suportes oferecidos pela ferramenta, diante de um cenário com número significativo de pessoas conectadas simultânea e sincronamente, a qualidade da transmissão em vídeo e áudio, assim como janelas de conversação por mensagens de texto compartilhadas (bate papo). Por fim, o quesito conectividade ainda é um problema para muitos brasileiros, sobretudo em localidades periféricas nas cidades, onde há precariedade na cobertura de sinal pelas operadoras de tele e web comunicação.

Diante das análises do grupo, as plataformas escolhidas e utilizadas para o desenvolvimento da pesquisa foram Google Meet, Skype e as chamadas de vídeo por WhatsApp. Estes respectivos aplicativos possuem um consumo baixo de dados de internet quando comparados a outros, todavia mais robustos e completos em suas funções. Com base nesses critérios as atividades de pesquisa, foram adaptadas à modalidade remota garantindo também a participação da comunidade externa na pesquisa, sem maiores complicações.

Os pesquisadores organizaram virtualmente o Cine Debate, tradicionalmente realizado na sala de cinema da universidade em formato presencial. O convite utilizou a web para divulgação (Facebook, Instagram) e o debate virtual foi aberto à sociedade. Os pesquisadores criaram um cartaz virtual utilizando o Canva, plataforma de edição gratuita e disponível on-line. A comunidade universitária foi convidada a assistir ao documentário "Mulheres das Águas", produzido pela FioCruz e a Universidade Federal do Rio de Janeiro [UFRJ] utilizando o link disponível no YouTube. Este documentário foi crucial para a pesquisa desenvolvida pelos alunos PIBIC, pois trata das principais dificuldades e processos relativos ao trabalho das marisqueiras em várias partes do Brasil, em especial no Nordeste.

No segundo momento ocorreu o debate para discutir aspectos sociais e legais contidos no documentário. A modalidade de cine debate virtual demandou especificidades como: escolha da plataforma considerando número de participantes; verificação prévia da qualidade de transmissão de vídeo durante o evento; divulgação da sessão online; captura de tela e gravação do evento para registro. Após os participantes assistirem ao documentário houve espaço para debates e aprofundamento do tema e das questões apresentadas no referido. $O$ evento cumpriu seus objetivos e serviu como subsídio informacional ao desenvolvimento do estudo, preservando suas características 
descritivas qualitativas, além de aproximar as ações extensionistas da universidade pública para diálogo com a sociedade civil.

Figura 1

Cartaz de Divulgação Cine Debate Virtual (2020).

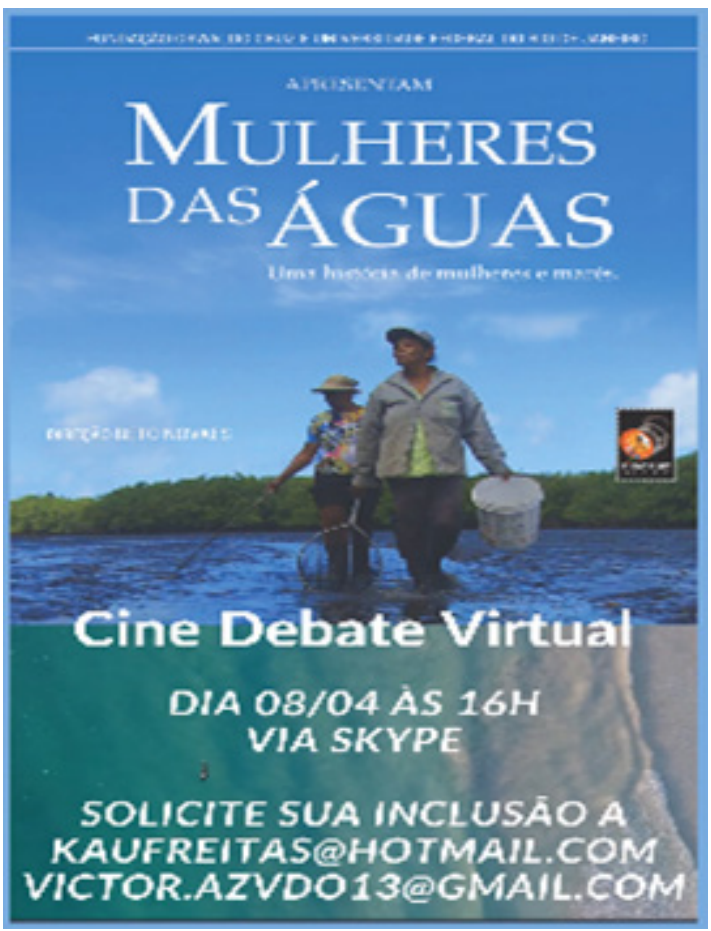

Nota: [FioCruz \& Universidade Federal do Rio de Janeiro (UFRJ). Adaptação nossa.]

Constataram-se dificuldades de alguns pesquisadores no quesito econômico, social e tecnológico para a realização do trabalho remoto e, também, no acesso à comunidade marisqueira da llha de Maré (Bahia). Em algumas ocasiões o único equipamento de acesso eram os telefones celulares com acesso à internet. Diante das impossibilidades físicas foram realizadas entrevistas on-line com uma advogada previdenciária e uma líder comunitária quilombola, via Skype e WhatsApp, respectivamente. As entrevistas tiveram duração de 60 minutos e todos os participantes puderam fazer arguições conforme o instrumento elaborado pelo grupo.

O padrão de organização dessas duas atividades foi: contato e convite, elaboração e aplicação de roteiro de entrevista, dinâmica de realização síncrona entre os participantes. Para realização das reuniões e demais encontros virtuais de prestação de contas das atividades desenvolvidas, os pesquisadores precisaram se apropriar de conhecimentos sobre as plataformas/meios utilizados. Foram feitos testes para compreender o uso da plataforma escolhida e sanar dúvidas dos alunos quanto aos modos de uso. Observou-se que a adaptabilidade às plataformas escolhidas ocorria rapidamente e à medida que estas eram utilizadas.

Os textos produzidos pelos pesquisadores em modo remoto foram: relatórios de atividades, roteiros de entrevistas, termos de autorização de imagem e texto, cartas-convite, cartas agradecimento, transcrições das entrevistas, textos da cartilha, editoração para formato livro digital, editoração de imagens utilizadas na cartilha (Figura 2). 
Figura 2

Capa da Cartilha elaborada pela equipe PIBIC (2020).

\section{Sou}

\section{marisqueira,}

\section{posso me}
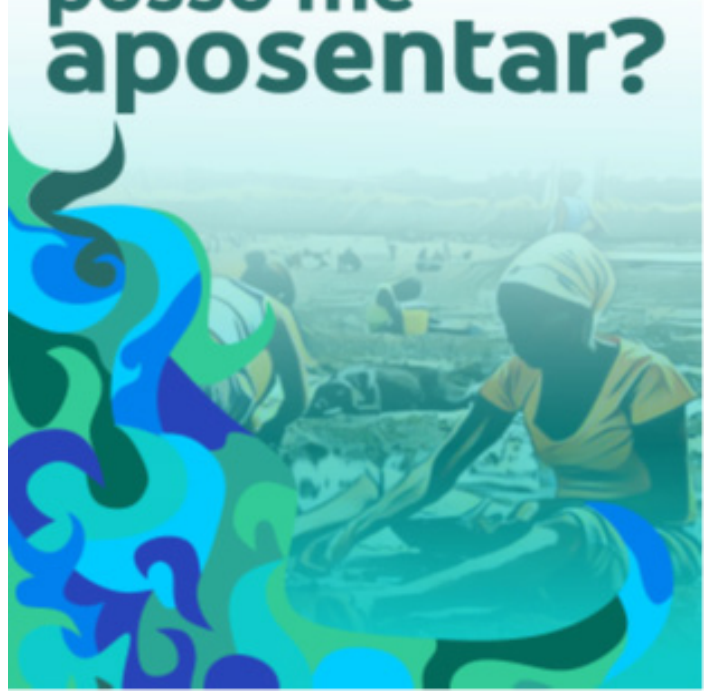

Nota: [Universidade Federal da Bahia (UFBA). Adaptação nossa.]

Os documentos, em geral, foram elaborados em editor de texto Word. Utilizamos o Google Documents como meio de contribuição coletiva aos textos produzidos a cada etapa da pesquisa e para correções on-line. Importante salientar que os pesquisadores utilizaram as plataformas de pesquisa e indexadores para levantamento de textos científicos que subsidiassem as leituras e o trabalho, a saber Google Acadêmico e SciELO.

Por conta da realização de atividades em ambiente virtual onde era possível realizar gravações, alguns elementos dos diálogos foram mais bem monitorados pelos pesquisadores. Pequenos ruídos comunicacionais foram decorrentes do espaço de fala e interrupções ou pelo conflito de vozes ao microfone, outros ruídos causados pelos acessórios (microfone do aparelho utilizado), aspectos esses que são dispensados fora do ambiente virtual. Nesse sentido, a necessidade de um roteiro também se tornou mais evidente, visto que o espaço de fala precisa de uma maior organização (tempo e objetivos) para evitar microfonia e outros fenômenos particulares do ambiente virtual.

A participação dos alunos em dois congressos científicos virtuais foi realizada também na modalidade remota, a saber: no Congresso Virtual UFBA, realizado de 18 a 28 de maio de 2020, onde os pesquisadores apresentaram vídeo pôster sobre resultados parciais da pesquisa PIBIC; e nas X Jornadas de Secretariado e Assessoria da Escola Superior de Tecnologia e Gestão de Águeda, Universidade de Aveiro, em Portugal, realizadas em 03 de junho de 2020, evento em que os pesquisadores trataram sobre suas performances em trabalho remoto, como relato de experiência. Ambos os eventos demandaram além dos textos científicos submetidos, a gravação de vídeos para serem disponibilizados em plataformas com canais específicos das instituições. Para a UFBA, a apresentação deveria ser somente na modalidade Webnário, disponível no YouTube sob o título: "As Mulheres, 
graduandas e o mar: rede documental e informacional para aposentadoria das marisqueiras" (YouTube, 2020). Para o vídeo, foram elaborados roteiro, ensaios, testes de cenário, luz, câmera e por fim, após a gravação houve editoração do material submetido à UFBA que, após aprovação da universidade, foi disponibilizado no canal da IES na referida plataforma.

Quanto ao evento da universidade portuguesa, embora tenha sido gravado um vídeo sob as mesmas condições de elaboração mencionadas, ele só seria utilizado em caso de impossibilidade de apresentação em modo síncrono. Ainda assim, importa destacar que a equipe de pesquisadores teve semelhante cuidado e trabalho com a elaboração de roteiro, da fala e tratamento do material de exibição (editoração) que foi totalmente criado e organizado pela equipe de pesquisadores e encaminhado para a organização do evento.

Por fim, os alunos conseguiram desenvolver as ações previstas no Projeto de Pesquisa da Iniciação Científica cumprindo os prazos e o cronograma proposto pela UFBA, desde que passaram a utilizar ferramentas de trabalho remoto na totalidade de seu planejamento e realização das tarefas. Com o trabalho remoto e os usos das tecnologias digitais foi possível concluir a pesquisa PIBIC a contento gerando: relatórios de pesquisa, pôsteres, cards, elaboração de cartilha eletrônica, elaboração de cartilha para impressão e participação em atividades síncronas e assíncronas que incluíram: reuniões, cine debate, entrevistas, participação em Congresso UFBA e encontro internacional de universidade portuguesa. Para todas as atividades descritas as técnicas secretariais foram imprescindíveis.

A seguir, dissertamos sobre as conclusões desta investigação.

\section{Considerações Finais}

O estudo teve por objetivo identificar as necessidades e o desenvolvimento do trabalho técnico dos pesquisadores juniores e como eles reestruturaram a metodologia das atividades de pesquisa de um projeto PIBIC adaptando-a para o trabalho na modalidade virtual.

Desse modo, este estudo evidenciou a necessidade de melhorar o desempenho e atividades de investigação remota realizadas pelos estudantes durante a pandemia da COVID-19, cujo perfil apontou demandas socioeconômicas e tecnológicas que interferem na performance para o trabalho remoto. Tratando-se de pesquisadores graduandos do curso de Secretariado Executivo da UFBA, coordenados e orientados por uma pesquisadora doutora e, também, Secretária Executiva, algumas plataformas e técnicas secretariais que já eram de domínio cotidiano dos sujeitos da pesquisa, mas integraram as atividades para consecução da pesquisa, mais especificamente em $100 \%$ do trabalho. Todas as rotinas de cunho administrativo e operacional da pesquisa, assim como as estratégias de pesquisa foram repensadas e reorientadas para a modalidade remota.

Com a pandemia, a impossibilidade de encontros presenciais e visitas ao campo da pesquisa (Ilha de Maré), os conhecimentos, técnicas secretariais e os meios virtuais foram imprescindíveis para a realização da Pesquisa PIBIC. O estudo observou, também, a necessidade da comunidade acadêmica de repensar os métodos tradicionais de pesquisa frente às adversidades do mundo contemporâneo. 
A pesquisa na modalidade virtual foi possível devido aos conhecimentos e aplicações das técnicas secretariais pelos pesquisadores PIBIC, além dos usos dos aplicativos e plataformas que possibilitaram a realização das atividades propostas no projeto da pesquisa, na modalidade de trabalho remoto. Diversas ações que estavam planejadas para ocorrer na modalidade presencial migraram para possibilidades virtuais, em veículos e espaços digitais já conhecidos dos pesquisadores PIBIC, que conseguiram fazer as adaptações necessárias em seus ritmos em adequações à nova modalidade de trabalho imposta pela pandemia da COVID-19.

Os aplicativos e plataformas mobilizaram o grupo de estudantes a conhecer mais e melhor suas aplicabilidades para a realização de atividades e eventos integralmente virtuais. Isso implicou em estudos sobre o tema e experimentações dos softwares e aplicativos a fim de determinar suas escolhas. Os alunos também precisaram melhorar seu desempenho no uso dos meios virtuais para o desenvolvimento das atividades de pesquisa de Iniciação Científica buscando informações sobre as plataformas e seus usos confirmando, assim, as perspectivas apontadas para o trabalho remoto pelos secretários executivos (Fanzeres \& Lima, 2018) e mesmo Matos (2016).

É preciso considerar também que alguns dos alunos tiveram implicações nos acessos às plataformas e aplicativos por questões econômicas, já que a maioria dos aplicativos necessita internet e incidem nos pacotes de dados aos quais os alunos pesquisadores, ressaltamos principalmente aqueles de baixa renda, têm acesso. Este item também incide sobre o acesso aos hardwares, já que nem todos os pesquisadores PIBIC têm notebook ou computadores domésticos. Em geral utilizam os computadores nos laboratórios localizados na Universidade, à qual não dispunham de acesso durante o isolamento e o encerramento das atividades presenciais na UFBA.

Conclui-se, portanto, a necessidade de ampliar o conhecimento sobre aplicativos, plataformas, softwares e hardwares que viabilizem o trabalho remoto e a comunicação no Teletrabalho. E, em nosso entender, para o teletrabalho que relacione pesquisa PIBIC da Universidade destacamos a necessária disponibilidade de acesso a computadores para os alunos que estejam comprometidos com a pesquisa universitária.

A pesquisa evidenciou a necessidade de investimentos e políticas públicas para suporte à Iniciação Científica, de modo a considerar o possível empréstimo de notebooks, a oferta de internet de alta velocidade e maior capacitação digital para os pesquisadores em situação de vulnerabilidade econômica social que participem desses programas, como o PIBIC.

Como sugestão para estudos futuros, julgamos que seja importante aprofundar as discussões sobre o trabalho remoto de pesquisadores iniciantes, com ou sem pandemia. Devem ser incluídas as técnicas secretariais e suas modalidades virtuais. Face às dificuldades de disponibilização de vacinas para COVID-19, é possível que esse cenário ainda faça parte do nosso cotidiano nos próximos meses e incida semelhantes dificuldades nas pesquisas PIBIC que seguem em modo remoto. 


\section{Referências}

Arroyo-Sanchez, A. S., (2020). Infodemia, la otra pandemia durante Covid-19. Recuperado em 02 junho, 2020, de https://preprints.scielo.org/index.php/scielo/preprint/download/367/456/455

Belluzzo, R.C.B., (2018). A competência em informação no Brasil: cenários e espectros. São Paulo: ABECIN.

Decreto Legislativo no 6, 20 de março de 2020 (2020). Reconhece, para os fins do art. 65 da Lei Complementar no 101, de 4 de maio de 2000, a ocorrência do estado de calamidade pública, nos termos da solicitação do Presidente da República encaminhada por meio da Mensagem no 93, de 18 de março de 2020. Brasília. 2020. Recuperado 20 abril, 2020, de http://www.planalto.gov.br/ccivil_03/portaria/DLG6-2020.htm

Decreto-Lei no 5.452, de 1o de maio de 1943 (1943). Aprova a Consolidação das Leis do Trabalho. Brasília. 1943.

Recuperado em 10 maio, 2020, de http://www.planalto.gov.br/ccivil_03/decreto-lei/del5452.htm

Fanzeres, N., \& Lima, L. (2018). Evidências da adaptação de profissionais de secretariado ao trabalho virtual. $R$. G. Secr., GESEC., 9(1), 42-64. Recuperado em 15 fevereiro, 2020, de https://www.revistagesec.org.br/ secretariado/article/view/634

Lei no 13.467, de 13 de julho de 2017 (2017). Altera a Consolidação das Leis do Trabalho (CLT), aprovada pelo Decreto-Lei no 5.452, de 1으 de maio de 1943, e as Leis n o 6.019, de 3 de janeiro de 1974, 8.036, de 11 de maio de 1990, e 8.212, de 24 de julho de 1991, a fim de adequar a legislação às novas relações de trabalho. Brasília. 2017. Recuperado em 10 agosto, 2020, de http://www.planalto.gov.br/ccivil_03/_ato20152018/2017/lei//13467.htm

Lei n. 13.979, de 6 de fevereiro de 2020 (2020). Dispõe sobre as medidas para enfrentamento da emergência de saúde pública de importância internacional decorrente do coronavírus responsável pelo surto de 2019. Brasília, DF. Recuperado 20 abril, 2020, de http://www.planalto.gov.br/ccivil_03/_ato2019-2022/2020/lei/ I13979.htm

Matos, R. S. F. (2016). Nômades Digitais: perfis, motivações e viabilidade. Dissertação de Mestrado, Fundação Getúlio Vargas, Escola Brasileira de Administração Pública e de Empresas, Rio de Janeiro, RJ, Brasil.

Medida Provisória no 927, de 22 de março de 2020 (2020). Dispõe sobre as medidas trabalhistas para enfrentamento do estado de calamidade pública reconhecido pelo Decreto Legislativo no 6, de 20 de março de 2020, e da emergência de saúde pública de importância internacional decorrente do coronavírus (covid-19), e dá outras providências. Diário Oficial da União. Brasília. 2020. Recuperado em 10 agosto, 2020, de https:// www.in.gov.br/en/web/dou/-/medida-provisoria-n-927-de-22-de-marco-de-2020-249098775

Nilles, J. (1973). Fazendo do teletrabalho uma realidade. São Paulo: Futura.

Portaria no 103, de 19 de março de 2020 (2020). Dispõe sobre a suspensão das atividades na

UFBA, devido à disseminação do novo coronavírus (COVID-19). Recuperado em 10 agosto, 2020, de http://www.ims. ufba.br/sites/ims.ufba.br/files/noticias/portaria_103-2020.pdf

Rosenfield, C. L., \& Alves, D. A. (2011). Autonomia e trabalho informacional: o teletrabalho. Dados, 54(1), $207-233$. https://doi.org/10.1590/S0011-52582011000100006 
Serviço Federal de Processamento de Dados. (2020). Serpro amplia trabalho remoto em todas as suas unidades pelo país. Recuperado em 20 maio, 2020 de https://www.serpro.gov.br/menu/noticias/noticias-2020/serproestende-trabalho-remoto

Sociedade Brasileira de Teletrabalho e Teleatividades. (2020). Pesquisa Home Office Brasil 2016 - Teletrabalho e Home Office, uma tendência nas empresas brasileiras. Recuperado em 20 março, 2020, de http://www. sobratt.org.br/index.php/11-e-12052016-estudo-home-office-brasil-apresenta-o-cenario-atual-da-praticano-pais-sap-consultoria/

Universidade Federal da Bahia. (2020). UFBA interrompe atividades por tempo indeterminado em combate ao coronavírus. Recuperado em 28 novembro, 2020, de https://coronavirus.ufba.br/ufba-interrompeatividades-por-tempo-indeterminado-em-combate-ao-coronavirus

YouTube (2020). As mulheres, graduandas e o mar: rede documental e informacional para aposentadoria das marisqueiras. Recuperado em 6 junho, 2020, de https://www.youtube.com/watch?v=tfxJ7_6mza4\&t=54s 